\title{
Analysis of foot and footprint measurements to determine the sex of an individual
}

\author{
Jayaweera $\mathrm{AS}^{1}$, Nandasena $\mathrm{T}^{1}$, Nawarathna $\mathrm{LS}^{2}$, Alahakoon $\mathrm{S}^{1}$, Ariyasinghe $\mathrm{S}^{1}$, \\ Nanayakkara $\mathrm{CD}^{1}$ \\ ${ }^{I}$ Department of Basic Sciences, Faculty of Dental Sciences, University of Peradeniya. \\ ${ }^{2}$ Department of Statistics and Computer Science, Faculty of Science, University of \\ Peradeniya. \\ *suchash@yahoo.com
}

Sex determination becomes a major problem to forensic experts especially when only fragments of the body are left following mass disasters. Sex determination is a major aspect in the identification of a person along with the estimation of age, stature and ethnicity. Analysis of skeletal biology is an integral part of this process. Previous studies done on human skeletons have shown population specificity in sexual dimorphism. Similar studies applicable to the Sri Lankan population are scarce. The present study is an attempt to use foot and footprint measurements for sex determination. A total of 116 (51 males and 65 females) Sinhalese individuals participated in the study. Three anthropometric measurements of the foot and seven linear measurements of the footprint were obtained from each individual for the analysis. All foot and foot print dimensions were significantly greater in males than in females $(\mathrm{p}<0.001)$. Univariate and stepwise discriminant function analyses performed to assess the sex classification potential of foot and footprint variables showed that overall cross-validated per cent correct classifications range from $73.9 \%-96.6 \%$ and $77.4 \%-94 \%$ for the right and left foot, respectively. The most dimorphic single parameter was the foot length providing cross validated accuracy rates of $96.6 \%$ and $94.0 \%$ for the right and left foot, respectively. The study shows that foot and foot print measurements can be used to classify sex with a high degree of accuracy among the Sinhalese population in Sri Lanka. 